\title{
Summary of Research on Related Technologies of Ship Dynamic Positioning System
}

\author{
Shuaiyang Wang, Xuanfang Yang, Zheng Wang*, and Liangkun Guo \\ School of electrical engineering, Naval University of engineering, 430033 Wuhan, China
}

\begin{abstract}
Dynamic positioning means that the ship can resist the influence of wind, wave, current and other environmental interference through its own propeller without anchoring, so as to realize the maintenance of its fixed position or expected track on the sea surface, with the advantages of not limited by water depth, rapid input or evacuation, good mobility and accurate positioning. This paper briefly introduces the components, working principle and mathematical model of the ship dynamic positioning system; On this basis, this paper summarizes the research status of the control strategy, filtering and data fusion, thrust distribution method and other related technologies of the ship dynamic positioning system at home and abroad; Finally, the development trend of the above-mentioned related technologies is discussed, so as to provide reference for the research in this field.
\end{abstract}

\section{Introduction}

With the development and progress of society, human consumption of energy grows with each passing day. While the energy resources on the mainland are being developed and applied, people gradually turn their attention to the marine field rich in natural resources, so the research on mooring mode of floating production system (including semi submersible platform and production tanker) for deep-sea operation is increasingly valued [1]. In general, the mooring system is mainly used in the floating production system, which has the advantages of simple structure, reliability and good economy. However, with the increase of water depth, the bottom grasping force of traditional mooring system decreases and the difficulty of anchoring increases. As the length and strength of the anchor chain also increases, resulting in the sharp increase of the weight of the ship, the offshore chain laying operation also becomes complex, so the cost and installation cost of the anchor chain increase sharply, and its positioning function is also greatly restricted. At the same time, with the more complex and changeable marine environment under the vast ocean, a set of new technology is more and more needed to be put into production [2]. Therefore, the research of dynamic positioning system (DPS), which is suitable for keeping the position constant or track stable in deep water, is more and more important.

\section{Theory of ship dynamic positioning system}

\subsection{Composition and working principle of dynamic positioning system}

DPS is mainly composed of three parts (as shown in Fig. 1): 1) measurement system, which measures the position and heading direction of the ship or floating platform relative to a certain reference point; 2) the control system calculates the disturbing force of the ship or floating platform according to the external environment interference (wind, wave and current), and then calculates the position and heading information obtained by the disturbance and measurement system to calculate the ship's fixed position 3) thrust system, which consists of several thrusters, provides thrust to counteract the external time-varying environmental load according to a series of thrust commands issued by the control system.

The working principle of the DPS is: it can resist the influence of wind, wave, current and other environmental interference through its own propeller, and automatically control the position and heading of the ship, so as to realize the maintenance of the fixed position or expected track of the ship or floating platform on the sea surface. When the dynamic positioning operation is implemented, the central processing unit ( the controller module shown in Fig.1) receives the data from various sensors for processing, and estimates the ship's position and heading direction. The estimated value is displayed on the console and constantly updated. When the automatic dynamic positioning mode is executed, the dynamic positioning

\footnotetext{
* Corresponding author:marchy618@163.com
} 
(DP) operator sets an expected set point, which can be the current position of the ship, a new position or a

moving target, such as remote operated vehicle (ROV).

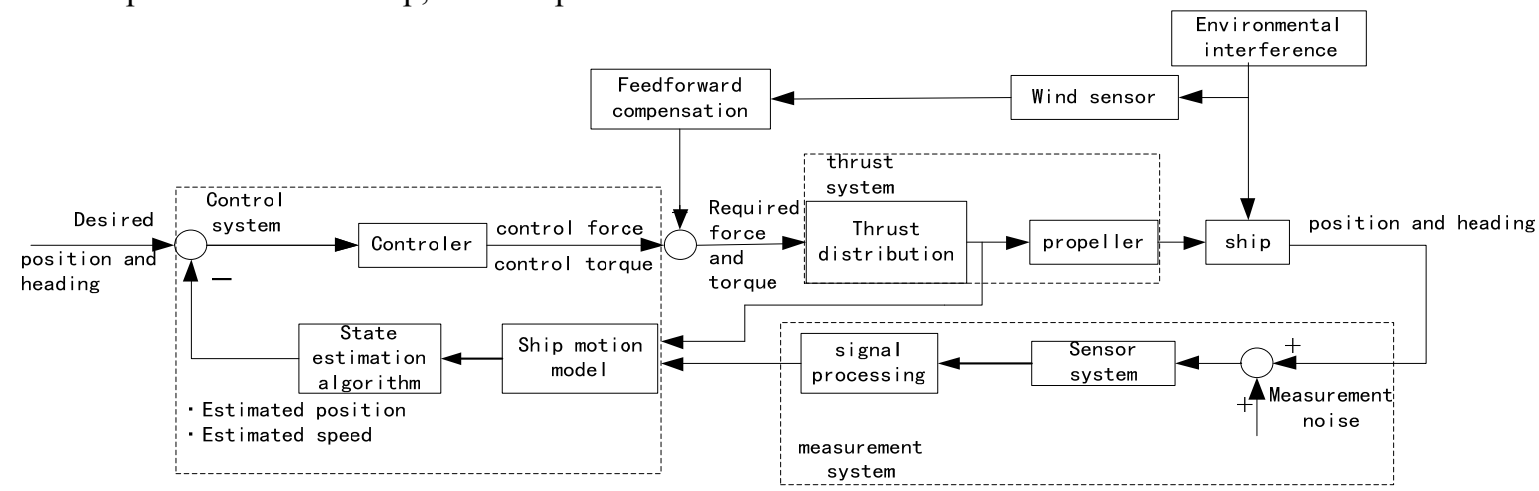

Fig.1. Composition of DPS

The CPU compares the estimated position and heading of the ship with the command set value to obtain the error value, and generates the propulsion command to reduce the error value. After reaching the set point, the system automatically compensates for wind, wave, current and other environmental factors to keep the ship stable at the set point. The DPS manufacturer created an accurate mathematical model of the ship at the design stage, which combines aerodynamic and hydrodynamic factors. Therefore, despite the disturbance of wind, wave and current, the control system can still keep the ship at the set point after calculating the thrust command.

\subsection{Dynamic positioning ship mathematical model}

The classical mathematical model of DP system is the six degree of freedom model provided by fossen [3]:

$$
\left\{\begin{array}{l}
\dot{\eta}=R(\psi) v \\
M \dot{v}+C_{R B}(v) v+C_{A}\left(v_{r}\right) v_{r}+D\left(v_{r}\right)+G(\eta)= \\
\quad \tau_{\text {wind }}+\tau_{\text {wave } 2}+\tau_{\text {moor }}+\tau_{\text {ice }}+\tau_{\text {thr }}
\end{array}\right.
$$

Where: $v=[u, v, w, p, q, r]^{T}$ is system state variable, $u$ is surge, $v$ is sway, $w$ is heaving, $p$ is roll angle, $q$ is pitch angle, $r$ is heading angle. Fixed point control, heading keeping and track tracking of surface ships are still the main control tasks of the existing DPS, and the control targets are longitude, latitude and heading angle of the ship. Therefore, most of the current scientific research work and mature products do not consider the above-mentioned complex six degree of freedom model, and only study the following simplified model [3-5]:

$$
\left\{\begin{array}{l}
\dot{\eta}=R(\psi) v \\
M \dot{v}=-D(v)+\tau+\Delta(t) \\
R(\psi)=\left[\begin{array}{ccc}
\cos \psi & -\sin \psi & 0 \\
\sin \psi & \cos \psi & 0 \\
0 & 0 & 1
\end{array}\right]
\end{array}\right.
$$

Where: $\eta=[x, y, \psi]^{T}$ is the state variable composed of surge, sway and yaw; $v=[u, v, r]^{T}$ is the state variable composed of longitudinal velocity, transverse velocity and heading angular velocity; $R(\psi)$ is the coordinate transformation matrix; the inertia matrix $M$ includes the hydraulic added mass; $D$ is the linear hydrodynamic damping coefficient; $\tau$ is the control force and moment.

This paper only introduces two models. In practice, such as unknown disturbance, input restriction, firstorder wave force, etc., are not to be studied here.

\section{Research progress of DP system control strategy}

The control strategy in the control system is an important part of the DPS in the development process, which represents the development level of the DPS. According to section 1.2 of DP ship mathematical model, DPS has nonlinear and coupling effect. At the same time, ship model parameter perturbation, sea state mutation and other factors will also reduce the operation accuracy of DP ship. Therefore, it is of far-reaching significance to study the control strategy of DPS.

DP control strategies can be roughly divided into three categories: the first category usually adopts the method of combining low-pass filter/notch filter with PID control law; the second category usually adopts the method of Kalman filter (KF) with optimal control, nonlinear control method and robust control method; the third category usually adopts the method of combining nonlinear observer with intelligent/hybrid control. For example, neural network control, fuzzy control, adaptive control, fault-tolerant control, model predictive control and so on. It should be noted that there is no obvious time limit between the second and third categories of control strategies, which are still under development.

1)The first major category of DP control strategy is the classical control strategy, namely PID controller. This controller is used to control the motion of the ship on the sea level in three degrees of freedom: surge, sway and yaw. The principle block diagram of common dynamic positioning PID control strategy is shown in Fig. 2 . 


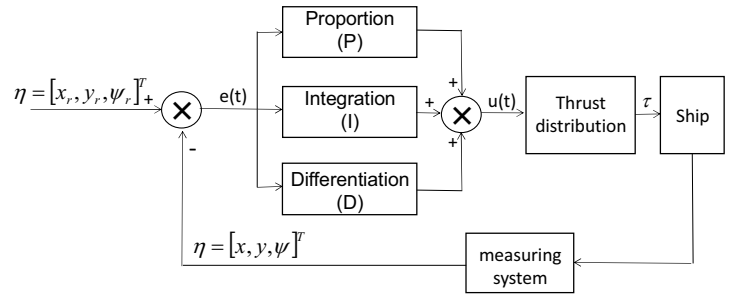

Fig.2. The Schematic diagram of PID control strategy for DP model

In the early stage, the application of PID controller in DP has achieved considerable success, but it also has an inevitable disadvantage, that is, it is difficult to select the appropriate PID parameters when designing the controller. In addition, because PID controller uses the linear combination of $\mathrm{P}, \mathrm{I}, \mathrm{D}$, and requires the model to be known accurately, while DPS is a complex nonlinear system, its control effect will be limited.

2)In view of the limitations of PID control, in order to meet the actual operation requirements, the second category of DP control strategy was generated and has been developed far-reaching [6-9]. The control strategy in this stage is composed of KF and optimal control. The specific method is as follows : KF is used to filter and estimate the ship's comprehensive motion position information, and the estimated low-frequency motion state is fed back to the controller. Thus, the DP control system only needs to compensate and control the lowfrequency motion caused by the disturbance, but does not respond to the first-order wave high frequency interference such as wave and noise. However, because there are many state variables and hundreds of state covariances in the system calculation, the amount of online calculation is very large, so many of them are difficult to adjust online.

Nonlinear control and robust control are also introduced into DPS. In reference [8-9], a nonlinear feedback controller is designed for the DPS by using backstepping (backstepping), which avoids the error caused by the linearization of the ship motion equation by the above KF under the assumed conditions. The disadvantage of backstepping method lies in the lack of systematic method in constructing Lyapunov function. In reference[10], a backstepping integral controller is designed for a ship model with uncertain time-varying environmental forces. In the design process of the controller, a two-step Lyapunov function is constructed to recursively deduce the control rate of ship motion, so that the ship can obtain stable control effect under bounded disturbance conditions. In reference [11-12], a controller of DPS is designed based on nonlinear sliding mode control, and it is proved that the designed controller has global stability. In reference [13], based on ADRC technology, a nonlinear DP controller for ships is designed. The controller estimates the speed of ship motion and the total disturbance of the system by a nonlinear observer, and uses nonlinear feedback to compensate for the DP control of the ship. The simulation results show that the controller has strong anti-interference ability and robustness.

3)In the third generation, the control law is designed by combining nonlinear observer with intelligent / hybrid control. Fuzzy logic systems (FLS) and neural network $(\mathrm{NN})$ open up a new way to solve the model uncertainty of complex system because of their unique universal approximation characteristics. In reference[14], using the powerful modeling ability of artificial neural network, the forces generated by wind, wave and current are adaptively fed back to the system as feedforward to resist the possible drift of ships in advance. The simulation results verify the effectiveness of the algorithm. In reference[15], the adaptive fuzzy control method is introduced, so that the parameters of the controller can be adjusted continuously during the positioning process to adapt to the changes of external environment. Professor Du Jialu's team in China has adopted FLS / NN adaptive control method and achieved many research results. In reference[16], a robust $\mathrm{NN}$ adaptive controller design method is proposed for systems with unknown external disturbances and uncertain parameters. It is proved by Lyapunov stability theory that the controlled system can track the given desired position and heading, and the tracking error converges, which can meet the control accuracy requirements of DPS and ensure that all adaptive parameters are bounded. References [17-18] respectively consider the control input saturation and control input constraints in practical engineering. The control strategies proposed in the above results have passed the Lyapunov stability proof and simulation verification. However, when designing the controller, it is necessary to assume that all state variables of the system are measurable, but the actual situation is just the opposite, the system state variables cannot be measured by sensors. In reference[19-20], an adaptive output feedback controller based on FLS/NN is designed by constructing a high gain state observer. The correctness and effectiveness of the method are proved by theory and simulation.

The control strategy based on model predictive algorithm is also introduced into DPS. Model predictive control (MPC) is also known as rolling time domain control. The optimal control flow is as follows: 1) obtain the measured value of system state at the current moment; 2) solve an optimal control problem to obtain the time domain control rate of predictive control; 3) take the first control value as the system input; 4) return to the first step at the next moment. The advantage of MPC is that the control input variables can ensure the optimal value of the system performance function composed of tracking error in the time domain of predictive control, and can effectively deal with the constraints of complex systems and realize complex target control. Therefore, MPC has been successfully applied to ship DP control system[21]. Wang Yuanhui et al.[22] introduced the linear model predictive control technology into the design of ship DP system. The input saturation problem was treated as the constraint condition of model predictive control, and the ship DP control under input saturation was realized

Fault tolerant control is another effective way to improve the security and reliability of the system in case of system failure, which belongs to software redundancy. At present, the fault-tolerant control of ship DP system 
mainly focuses on the design of DP control system for propulsion system and sensor failure [23-24]. Ning Jipeng [25] designed a control reconfiguration method based on virtual sensor, which solved the DP problem of several kinds of sensor failures. In reference [26], an adaptive sliding mode controller is designed which is independent of fault detection module and upper and lower bounds of fault information. Fault tolerant control of DP ship is a very complex subject. Although the faulttolerant control for thruster and sensor fault has made a lot of achievements, the method of fault-tolerant control of ship is not perfect and needs further research.

\section{Research Progress on thrust distribution of DP thrust system}

In foreign reference[27-29], an unconstrained quadratic thrust allocation method is proposed. Although the thrust allocation problem is transformed into an unconstrained quadratic optimization problem, the methods in reference [27-29] all consider the treatment of singularity, which shows that the method can avoid the singular structure of thrust allocation. Reference [27] solved the problem of positive and negative thrust generated by rotary thrusters. In reference [28], the thrust direction is calculated by the expected generalized force of a low-pass filter, and the singularity is solved by using the damped least square method when the thrust is calculated. In reference [29], a variety of unconstrained secondary thrust allocation methods for fixed multi thrusters are proposed, and the thrust allocation under failure of one of the thrusters is also considered.

In China, Wu Xianfa and Wang Yanying [30] used sequential quadratic programming to solve the thrust allocation problem of DPS, which can reduce fuel consumption and avoid singular structure. Yang Shizhi and Wang Lei et al.[31]proposed a pseudo inverse method for DP thrust allocation. The effectiveness of the proposed method was verified by an example. Shi Xiaocheng et al.[32] proposed the idea of combined bias and designed an adaptive combination bias strategy. The algorithm is based on the energy optimization method and can adaptively adjust the offset amount.

\section{Research progress of filtering and data fusion technology for dynamic positioning measurement system}

The information accuracy and credibility of DP measurement system is very important. Most of the dynamic positioning ships are equipped with a variety of position reference systems and sensors. During the positioning process, the ship is disturbed by wind, wave, current and other external factors, which makes the sensor polluted by high-frequency noise. Therefore, it is necessary to study the filtering technology of measurement system. Wang Zongyi et al.[33]used the $\mathrm{KF}$ to calculate the low-frequency motion of the ship with three degrees of freedom, which can also filter out the high-frequency noise in the comprehensive position information of the ship. Wang Xiaosheng et al. [34] combined the optimal control and adaptive Kalman filtering technology to estimate the high and low frequency motions of ships working in complex and changeable marine environment, so as to ensure that the positioning system only controls the low-frequency motion of ships. Extended Kalman filter (EKF) and unscented Kalman filter (UKF) are the improvement of Kalman filter. They are a kind of nonlinear filtering methods. Scholars at home and abroad apply them to ship state observer design, which can solve the linearization error caused by KF. In view of the shortcomings of KF and the nonlinear characteristics of ship mathematical model, Grimble [35] applies EKF to ship motion state estimation by calculating Jacobian matrix of nonlinear state transition function, and its effectiveness is verified by simulation. Jiang Fan et al. [36] proposed a model predictive EKF algorithm. When the system model noise is unknown, the system process noise variance is corrected in real time. The experimental results show that the filtering effect of this method is obviously better than that of traditional EKF. UKF approximates the probability density distribution of nonlinear function by UT transformation, and does not ignore the high-order term, which overcomes the defects of low accuracy and poor stability of EKF estimation. Jayasiri et al. [37] constructed UKF to estimate ship motion state and unknown time-varying ocean disturbance. Simulation results verify its effectiveness. However, when the state noise changes due to the complex marine environment or inaccurate model, the filtering algorithm can not adjust the noise parameters adaptively, and the filtering effect will be greatly affected. In this regard, Deng et al. [38] proposed a novel adaptive unscented Kalman filter (AUKF) is applied to DPS with time-varying noise statistical model uncertainty, model mismatch and drift force changing slowly. The covariance of measurement noise is adjusted based on residual covariance matching method, and then the covariance of process noise is adjusted by adaptive scaling factor. Simulation results show that the proposed AUKF can effectively improve the estimation accuracy and stability, so that the controller obtains better control performance, but this method can not fundamentally solve the non positive definite problem of process noise.

The sliding mode variable structure observer is also used in the design of state observer for DP. The sliding mode variable structure observer has strong robustness for nonlinear systems with uncertain disturbances and unmodeled dynamics, and can be used to solve the problem of unknown external environmental forces on DPS [39]. Under the condition that the parameters of the mathematical model of ship motion are uncertain and the ocean disturbance is time-varying unknown, Tannuri et al. [40] designed a DP robust nonlinear control law using sliding mode control method. The experimental results of a 1:150 scale model ship show its effectiveness. Xie Wenbo et al. [41] designed an adaptive sliding mode observer to estimate the ship speed and then designed a DP robust output feedback control law.

Liu Furong et al.[42] Studied the application of rolling time domain filtering method in the design of DP 
ship control system. In order to overcome the shortcomings of KF which requires accurate dynamic model and white noise, Li Lanhua et al. [43] Used the $\mathrm{H} \infty$ filtering method to obtain the low-frequency motion of the ship. Fu Mingyu et al. [44] established the mathematical model of ship motion at low frequency and high frequency, and designed the optimal estimation filter and finite impulse filter for filtering the high frequency component in the deviation signal. Qi guopeng [45] divides the dredger in the DP process into two states: free navigation state and variable draft operation state. In the condition of free navigation, the sage-Husa adaptive filtering algorithm is introduced; under the condition of variable draft, the improved adaptive filtering algorithm combining sage Husa adaptive filter and strong tracking KF is adopted, which solves the filtering problem in DP system. Xin Hong-jie [46] adopts adaptive linear neural network filter to eliminate the high frequency component of the ship effectively.

\section{Summary and Prospect}

This paper summarizes the research progress of controller design, thrust allocation method, filtering and data fusion in ship DPS.

1) The basic research of DP in China started later than that in foreign countries, and it is still backward. However, in recent years, it has shown a trend of catching up with and surpassing the international advanced level.

2) The controller design of control system is the core of DP. The development of control theory promotes the development of DP controller design. Many new results of control theory have been applied to DP system. Furthermore, the combination of multiple control strategies is being used by researchers to design DP controllers with dynamic uncertainties. In the future, the research of DP control system will develop towards the direction of compound, faster, more accurate and more robust.

3) The thrust distribution methods in thrust system mainly include deterministic method and intelligent optimization method. In order to improve the speed of thrust allocation strategy based on intelligent optimization algorithm, the research direction of thrust system is to study hybrid intelligent thrust allocation theory and strategy.

4) Kalman filtering method is still the mainstream method of measurement system filtering technology, so it is imperative to study more efficient filtering method of sensor noise in DPS. The research on data fusion technology in DP is less in China. We should learn from foreign data fusion technology and methods, and further apply it to DPS.

\section{References}

1. X. Q. Bian, M. Y. Fu, Y. H. Wang. Ship dynamic positioning. Beijing:Science Press, 2011:5-7.
2. Zhang $\mathrm{W}$ J, Liu $\mathrm{T}$ et al. Simulation study on dynamic characteristics of towed array for fourdimensional exploration of marine resources[J]. Ocean Engineering,2012,30(4):118- 124.

3. Fossen T I. Marine Control Systems: Guidance, Navigation and Control of Ships, Rigs and Underwater Vehicles[M]. Trondheim : Marine Cybernetics AS, 2002.

4. J. L. Du, Y. YANG. A robust adaptive neural networks controller for maritime dynamic positioning system[J]. Neurocomputing, 110 (2013): 128-136.

5. X. Hu, J. L. Du, J. W. Shi. Adaptive fuzzy controller design for dynamic positioning system of vessels[J]. Applied Ocean Research, 53 (2015): 4653.

6. BALCHEN J G,et al. A dynamic positioning system based on Kalman filtering and optimal control [J]. Modeling, Identification and Control, 1980,1 (3): $135-163$.

7. GRIMBLE M J, PATTON R J, WISE D A. The design of dynamic positioning control systems using stochastic optimal control theory [C].OCEANSU USA :San Diego, 1979 :488 - 497.

8. FOSSEN T I,GRVLEN A. A tutorial on nonlinear backs-topping : applications to ship control [J]. Modeling, Identification and Control,1999,20 (2):83 -135 .

9. LORIAL A. FOSSEN T I, PANTELEY E. A separation principle for dynamic positioning of ships : theoretical and experimental results $[\mathrm{J}]$. IEEE Transactions on Control Systems Technology ,2000,8 (2):332-343.

10. Fossen T I, Grovlen A. Nonlinear output feedback control of dynamically positioned ships using vectorial observer backstepping. IEEE Transactions on Control Systems Technology, 1998, 6(1):121128.

11. Tannuri E A, Agostinho A C, Morishita $\mathrm{H} \mathrm{M}$ et al. Dynamic positioning systems: an experimental analysis of sliding mode control. Control Engineering Practice, 18(10), 2010:1121-1132.

12. W. B. Xie, M. Y. Fu, X. C. Shi. Design of Adaptive Sliding Mode Passive Observer for Dynamic Positioning Ships. Control Theory and Application, 2013, 30(1): 131-136.

13. D. W. Zhao, X. Q. Bian, F. G. Ding. Design of nonlinear ship dynamic positioning controller[J]. Journal of Harbin Engineering University, 2011, 32(01): 57-61.

14. L. Ding, X. G. Mao. Dynamic positioning of ships using a planned neural network controller $[\mathrm{J}]$. Journal of Ship Research, 1996,40(2) : 164 - 171.

15. S. M. Rui, J. M. Zhu, G. Y. Huang. Ship dynamic positioning using adaptive fuzzy control. Journal of Shanghai Jiaotong University, 2000, 34 (1): 56-59.

16. J. L. Du, Y. YANG. A robust adaptive neural networks controller for maritime dynamic 
positioning system[J]. Neurocomputing, 110(2013): 128-136.

17. J. L. Du, X. Hu. Robust dynamic positioning of ships with disturbances under input Saturation[J]. Automatica, 2016, 73: 207-214.

18. X. Hu, J. L. Du. Robust adaptive NN control of dynamically positioned vessels under input constraints[J]. Neurocomputing, 2018, 14: 1-12.

19. X. G. Lin, J. Nie, Y. Z. Jiao, et al. Nonlinear adaptive fuzzy output-feedback controller design for dynamic positioning system of ships[J]. Ocean Engineering, 2018, 158: 186-195.

20. J. L. Du, X. Hu. Adaptive robust output feedback control for a marine dynamic positioning system based on a high-gain observer[J]. IEEE Transactions on Neural Networks and Learning Systems, 2015: 112.

21. ASMUND V. F.. Dynamic positioning by nonlinear model predictive control[D]. Norwegian University of Science and Technology, 2008.

22. Y. H. Wang, X. C. Shi, X. Q. Bian. Ship dynamic positioning constraint control based on model predictive control $[\mathrm{J}]$. Shipbuilding Engineering, 2007, 29 (3): 22-25.

23. FLAVIA B.,GIANLUCA I., SAURO L., et al. Advanced control for fault-tolerant dynamic positioning of an offshore supply vessel[J]. Ocean Engineering, 2015: 472484.

24. M. Y. Li. Research on fault tolerant control method of dynamic positioning ship propeller [D], Harbin: Harbin Engineering University, 2018.

25. J. P. Ning. Research on fault tolerant control method of ship dynamic positioning [D]. Harbin: Harbin Engineering University, 2013.

26. Hao Liying, Han Jincheng, Guo Ge, et al. Robust sliding mode fault tolerant control for ship dynamic positioning system with thruster failure $\mathrm{M}$. control and decision making, 2019:1-7.

27. SORDALEN O J. Optimal thrust allocation for marine vessels D].Control Engineering Practice, 1997 (5) :1223 -1231.

28. BERGE S P,FOSSEN T I. Robust control allocation of ove-ractuated ships : experiments with a model ship [C] . IFAC Conference on Maneuvering and Control of Marine Craft, Brijuni, Croatia, 1997 : $193-198$.

29. GARUS J. Optimization of thrust allocation in the propulsion system of an underwater vehicle [J]. International Journal of Applied Mathematics and Computer Science ,2004,14 (4) :461-467.

30. X. F. Wu, Y. Y. Wang. Design of the thrust allocation for the dynamic positioning system D] . Ship and Ocean Engineering,2008,37 (3) :92 -96.

31. S. Z. Yang, L. Wang, P. Sun.Optimal thrust allocation logic design of dynamic positioning with pseudoinverse method [J]. Journal of Shanghai Jiaotong University (Science) ,2011,16(1) :118-123.
32. X. C. Shi, Y. S. Wei, J. P. Ning, et al. Research on group biasing thrust allocation algorithm based on power optimization $[\mathrm{J}]$. Shipbuilding of China,2012,53 (2) :96-104.

33. Z. Y. Wang, X. Zhong, et al. Mathematical models and filtering methods for dynamic ship positioning $[\mathrm{J}]$. Journal of Marine Science and Application, 2002,23 (4):24-28.

34. X. S. Wang. Design and test of dynamic positioning system [J] . Shipbuilding of China,1991 (3):11 - 19.

35. GRIMBLE M J .The Design of Dynamic Positioning Control Systems Using Stochastic Optimal Control Theory [J]. Optimal Control Applications and Methods, 1980,1(2): 167-202.

36. J. Fan, H. X. Xu, H. Feng, W. G. Yu, W .J. Li , Y. H. Chen. Dynamic positioning state estimation based on model predictive extended Kalman filter [J]. Shipbuilding Engineering, 2019,41 (07): 86-91 +132 .

37. Jayasiri A, Nandan A, Iratiaz S, et al. Dynamic Positioning of Vessels Using a UKF-Based Observer and an NMPC-Based Controller. IEEE Transactions on Automation Science \& Engineering, 2017,14(4):1778-1785.

38. F. Deng , H. L. Yang, L. J. Wang, et al. UKF Based Nonlinear Offset-free Model Predictive Control for Ship Dynamic Positioning Under Stochastic Disturbances[J]. International Journal of Control Automation and Systems, 2019, 17(3).

39. OLE M. R. R.,ASTRID H. B.,MORTEN B.. Comparing controllers for dynamic positioning of ships in extreme seas[C]. IFAC-PapersOnLine 4923 (2016) 258-264.

40. Tannuri E A, Agostinho A C, Morishita H M et al. Dynamic positioning systems: an experimental analysis of sliding mode control. Control Engineering Practice, 18(10), 2010:1121-1132.

41. W. B. Xie, M. Y. Fu, X. C. Shi. Design of adaptive sliding mode passive observer for dynamic positioning ship $[\mathrm{J}]$. Control theory and application, 2013, 1 (30): 131-136.

42. F. R. Liu, H. Chen, H. B. Gao. Application of moving horizon filter for dynamic positioning ship [J]. Journal of Wuhan University of Technology,2010, 32 (12): 117-120.

43. L. H. Li, G. Q. Xia. Application of $\mathrm{H} \infty$ filtering dynamical positioning of the ship $[\mathrm{J}]$. Techniques Automation and Application, 2005,24 (11) : 35-37.

44. M. Y. Fu, F. G. Ding. The design and study of filter in ships dynamic positioning system $[\mathrm{J}]$. Ship Engineering, 1996(2) :43-46.

45. G. P. Qi. Ship motion modeling and filtering technology research on trailing suction hopper dredger [D] . Zhenjiang: Jiangsu University of Science and Technology,2012.

46. H. J. Xin. Research on method of filtering and position controlling for a dynamic positioning ship [D] . Harbin Engineering University,2011. 